\title{
Editorial.
}

\section{Forensic Nuclear Medicine}

\section{Saad, I.}

Assistant professor of Nuclear Medicine Physics, Faculty of Medicine, Cairo University, Egypt and Chairman of Nuclear Medicine Technology Department-Inaya Medical CollegesRiyadh- KSA.

\section{Forensic Nuclear Medicine:}

The Forensic Medicine is defined as the application of medical knowledge to the investigation of crime, particularly in establishing the causes of injury or death. It is also known as the application of medical knowledge to the service of the law and the administration of justice. The problem of determining the cause of sudden or accidental death is one of the most important functions of Forensic Medicine ${ }^{(\mathbf{1})}$.

This expression has emerged recently after the need of using functional imaging data and results to help forensic physicians to solve criminal or judgmental cases investigations.

The combination of forensic and nuclear medicine results in the term Forensic Nuclear Medicine.

The supplementary role of functional imaging by nuclear medicine investigations has been demonstrated in the vast field of clinical medicine as nuclear medicine has become a routine examination of different pathologies.

Many traumatic lesions are often having internal manifestations which might be overseen by clinical examination. Morphological investigation as by $\mathrm{x}$-ray, sonography or computed tomography (CT) is often used for evaluation of these cases. However, these anatomical imaging methods may fail to delineate injuries, as has been shown in cases of bone lesions. The supplementary role of functional imaging by nuclear medicine investigations has been demonstrated as a routine examination of different transarctic lesions ${ }^{(2,3)}$.

Forensic Nuclear Medicine as also involved with cases having a radioactive administration in these situations there will be different precautions that should be taken into considerations, in the following different situations that may encounter the forensic physician ${ }^{(4)}$. 
1- Nuclear Medicine in some cases more important than

\section{Diagnosis of Forensic Medicine}

Forensic investigations with radiographic examinations have a vital role for forensic physicians. There is a potential role of radionuclide cerebral blood flow studies in forensic medicine and the under-utilization of the method at the time of incidence is criticized. Trauma to the head can cause functional disturbance rather than fractures or hematomas which can be diagnosed by radiographic tools; in this case functional imaging is mandatory to investigate this functional disturbance. Anosmia following head trauma may present a forensic issue with an important legal impact, especially in our Islamic countries with Shariaa law. Few studies have been published assessing the validity of brain SPECT before and after olfactory stimulation. It was also emphasized that functional imaging should be interpreted together with such other findings as psychometric tests and other neuroimaging methods $(5,6$, and 7 ).

As regard to the musculoskeletal imaging, functional imaging can provide more insight findings regarding the extent of the traumatic lesions and their functional consequences. Functional disturbances following traumatic events could be in morphological abnormalities. Targeted scintigraphic examinations can be applied for visualization of traumatic lesions or evaluation of functional disturbances caused by traumatic events ${ }^{(8)}$.

Also, a blunt trauma to the abdomen may cause functional damage to the internal structures such as the kidneys in which the undiagnosed damage may be figured out by the functional imaging tools such as renal scan. This may have impact on the reports of the forensic physician in judgmental situations and may alter the discussion of the judge.

These examinations can have a forensic impact and be entered as evidence in the courtroom in addition to conventional radiological imaging ${ }^{(9)}$.

\section{2- Nuclear Medicine Radionuclide Therapy and Forensic Medicine:}

a) Cardiac or respiratory arrest or transfer of therapy patient for medical reasons.

Resuscitation of patients containing radioactive material for radiotherapy or therapeutic nuclear medicine purposes poses special problems. 
The general principles to be followed in all cases are that the patient's welfare is paramount and possible radiation exposure to staff should not be considered a barrier to resuscitation, as, for the short times involved, the potential radiation exposure will be small.

\section{Attention should be paid to the}

\section{following points:}

- Notify the Department of Nuclear Medicine immediately.

- Staff involved in resuscitation should wear disposable gloves.

- Do NOT apply direct mouth-to-mouth resuscitation.

- Materials that have come into direct contact with the patient should, as far as is practicable, be kept to one side for examination by nuclear medicine staff. This particularly applies to airways, masks, endotracheal tubes, etc.

\section{b) Transfer to ICU or the CCU}

Attention should be paid to the following points:

If a transfer is required, the fact that the patient may still contain radioactive material should not interfere with the management of the case.

In the case of patients treated with 131I for whom intubation, catheterization or use of a nasogastric tube may be necessary, staff should wear gowns and gloves when handling the patient. Urine, gastric contents or other body fluids should be contained as far as possible by means of absorbent pads, and the pads held in a contaminated waste bag for treatment by nuclear medicine staff. Any suction bottles or urine bags used must not be discarded until checked for contamination ${ }^{(\mathbf{1 0})}$.

\section{c) Examination of staff involved in} resuscitation or handling of the patient

Staff who have been directly involved with the patient will need, for their own safety and peace of mind, to be assessed as to their potential radiation exposure, however small. This will include the best possible estimation of radiation exposure, and, where 131I is involved, administration of Lugol's iodine as soon as possible to block thyroid uptake of any absorbed contaminant, if necessary, and subsequent measurement of any thyroidal accumulation of 131I ${ }^{(11)}$.

The precautions done following the death of a therapy patient. All procedures should be put in place for the safe disposal of the bodies of patients who have received therapeutic doses. This may include labeling, contamination avoidance and notification of the staff that may have to handle the body. 
These procedures will obviously depend on the radiopharmaceutical involved, the dose and the time since administration ${ }^{(11)}$. The nuclear medicine department must be notified immediately after a death.

Only a minimum of laying out procedures should be attempted (e.g. the sheet in which the body is wrapped should be clearly labeled as containing a radioactive body). The label should be clearly visible to all those handling the body. The body should be removed from the ward to the mortuary.

The body should be removed from the ward to the mortuary as soon as possible after death, without attempting to remove any of the radioactive material, and placed in, if possible, the center section of the body storage refrigerator. This is to minimize any radiation exposure to staff who may be working in the mortuary. Consulting with nuclear medicine staff for advice on radiation safety and removal of highly contaminated tissues such as the thyroid $^{(11,12)}$.

\section{d) Autopsies and its precautions:}

If a corpse contains less than $(12 \mathrm{mCi})$ of 131I, the procedures normally observed during the autopsy are adequate for the examination unless such examinations are carried out frequently.
If, however, a corpse contains radioactivity in excess of the levels given above, the pathologist should be informed of the radiation levels likely to be encountered and of the hazards involved.

The methods employed and the precautions adopted should be chosen accordingly. Any hazards to persons involved in these operations or the need for compliance with international transport regulations depend on several factors relating to the nature of the radioactive sources.

In most instances, the issue is resolved by keeping the corpse in appropriate cold storage until twenty half-lives of radioactive decay have passed. If it is known that the radioactive material used for treatment will be selectively absorbed in a particular organ, for example, 131I in the thyroid, the organ should be excised before the examination proceeds and removed from the work area. It may later be disposed with the body ${ }^{(13,14)}$.

\section{Awareness about forensic nuclear} medicine: Due to the vital role of nuclear medicine in solving forensic cases, there should be methods for increasing the awareness of forensic physicians about the uses of functional imaging by the following means: 
1- Establishment of joint scientific teams with the forensic physicians to study different cases using functional imaging modalities.

2- Establishment of workshops that explore the role of nuclear medicine and functional imaging in forensic medicine.

3- Inviting the forensic physicians to attend nuclear medicine conferences and participate effectively in these events.

\section{CONCLUSION:}

The use of radioactive materials in patients has a lot of precautions that should be taken into considerations especially

\section{REFERNCES:}

1. Kevin Lothridge, Frank Fitzpatrick," Crime Scene Investigation, A Guide for Law Enforcement", National Forensic Science Technology Center, 2013.

2. Mirzaei S, Knoll P, Lipp RW, Wenzel T, Koriska K, Köhn H. Bone scintigraphy in screening of torture survivors. Lancet 1998; 352:949-51.

\section{Lök V, Tunca M, Kumanlio ğglu K,}

Kapkin K, Dirik G. Bone scintigraphy as clue to previous torture. Lancet 1991; 337:846-7. when the patients fall under critical situations such as cardiac arrest and in need of resuscitation. In other cases, these patients may die in the nuclear medicine unit or during isolation after therapy doses; in that case, a special procedure should be followed by the forensic physicians and by the staff responsible for the body transfer and storage. Some nuclear medicine investigations, such as brain imaging and bone and renal scintigraphy may have an additional impact in the evaluation of traumatic lesions and their functional consequences and could be applied more frequently in forensic cases.

4. Sang-Geon Cho, Jahae Kim, and Ho-Chun Song, Radiation Safety in Nuclear Medicine Procedures, Nucl. Med. Mol. Imaging. Mar; 51(1): 11-16; 2017.

5. Mirzaei S, Rodrigues M, Koehn H, Knoll P, Bruecke T. Metabolic impairment of brain metabolism in patients with Lowy body dementia. Eur. J. Neurol. 10:573-5; 2003.

6. Piskunowicz M, Lass P, Krzyzanowski M, Studniarek M. The usefulness of CBF brain SPECT in forensic medicine. A description of four cases. Nucl. Med. Rev. Cent. East. Eur. 4:47-50; 2001. 
7. Piskunowicz M, Krzyzanowski M, 11. ICRP. ICRP publication 73: Lass $\boldsymbol{P}$, Bandurski $\boldsymbol{T}$. The usefulness of radiological protection and safety in CBF brain SPECT in forensic medicine: medicine. Ann. ICRP. 26 (2); 1996.

the civil law code cases. A description of four cases. Nucl. Med. Rev. Cent. East. Eur.6:45-7; 2003.

\section{Altun G, Durmus-Altun G.} Confirmation of alleged falanga torture by bone scintigraphy - case report. Int. J. Legal. Med. 117:365-6; 2003.

9. Kalantarinia $\boldsymbol{K}$. Novel imaging techniques in acute kidney injury. Curr. Drug Targets. 10:1184-9; 2009.

10. ICRP publication 94: release of patients after therapy with unsealed radionuclides. Ann. ICRP. 34 (2); 2004.
12. Bohdan Bybel, Michel Blais, Richard Vandierendonck and Albert A. Driedger, Radiation Safety When a Patient Dies After Therapy, J. Nuc. Med. Techno. 26:206-207; 1998.

13. ICRP publication 106: radiation dose to patients from radiopharmaceuticals addendum 3 to ICRP publication 53. Ann. ICRP. 38 (1-2); 2007.

14. Mahmoud Hassan Shabon, "Proposed Regulations for Handling Radioactive Cadaver" 4th Int. Con. Rad. Res. Appl. Sci., Taba, Egypt. PP. 159: $163 ; 2014$. 